\title{
On Some Algebraic and Operator-Theoretic Properties of $\lambda$-Toeplitz Operators
}

\author{
Mehdi Nikpour \\ Department of Science and Mathematics, The American University of Afghanistan, Darulaman Road, Kabul, Afghanistan \\ Correspondence should be addressed to Mehdi Nikpour; mnikpour@auaf.edu.af
}

Received 4 September 2014; Revised 16 December 2014; Accepted 17 December 2014

Academic Editor: Dashan Fan

Copyright (C) 2015 Mehdi Nikpour. This is an open access article distributed under the Creative Commons Attribution License, which permits unrestricted use, distribution, and reproduction in any medium, provided the original work is properly cited.

Based on a spectral problem raised by Barría and Halmos, a new class of Hardy-Hilbert space operators, containing the classical Toeplitz operators, is introduced, and some of their Toeplitz-like algebraic and operator-theoretic properties are studied and explored.

\section{Introduction}

All of the work I am about to describe takes place in the Hardy-Hilbert space of the unit circle $\partial \mathbb{U}$, denoted by $H^{2}(\partial \mathbb{U})\left(=H^{2}\right)$, and consists of all square-integrable (with respect to the normalized arc-length measure $d \theta / 2 \pi$ ) functions, on $\partial \mathbb{U}$, whose negative Fourier coefficients all vanish; that is,

$$
\begin{aligned}
& H^{2}=\left\{f \in L^{2}(\partial \mathbb{U}) \mid\right. \\
& \left.\quad \widehat{f}(n):=\frac{1}{2 \pi} \int_{0}^{2 \pi} f\left(e^{\imath \theta}\right) e^{-i n \theta} d \theta=0, n \in \mathbb{Z}^{+}\right\} .
\end{aligned}
$$

For more details and basic properties of Hardy spaces, the reader is referred to [1, Chapters 1 and 2] or [2, Chapter 17].

Two of the most intensely studied classes of bounded operators on $H^{2}$ are Toeplitz and Hankel operators. Originally, an infinite matrix is called Toeplitz (resp., Hankel) if its entries depend just on the difference (resp., the sum) of their indices. Hence, Toeplitz matrices are the ones with constant diagonals, and Hankel matrices are those with constant skew-diagonals. They both play a decisive role in a very wide circle of problems in operator theory, $C^{*}$ algebras, moment problems, interpolation by holomorphic or meromorphic functions, inverse spectral problems, orthogonal polynomials, prediction theory, Wiener-Hopf equations, boundary problems of function theory, the extension theory of symmetric operators, singular integral equations, models of statistical physics, and many others. Also, there exists a vast literature on the theory of Toeplitz and Hankel operators; see, for example, [3-8].

Maybe a naive reason also for their importance is the fact that Toeplitz and Hankel operators are compressions of (bounded) multiplication operators and their flipped, respectively, to $H^{2}$. Indeed, any essentially bounded function $\phi$ on $\partial \mathbb{U}$ induces, in a natural way, three bounded operators, one on $L^{2}(\partial \mathbb{U})$ and the two others on $H^{2}$, as follows.

(i) The Multiplication operator $M_{\phi}$ is given by $M_{\phi} f=\phi f$, for $f \in L^{2}(\partial \mathbb{U})$.

(ii) The Toeplitz operator $T_{\phi}$ is defined, in terms of the orthogonal projection $P$ from $L^{2}(\partial U)$ onto $H^{2}(\partial U)$, as the compression of $M_{\phi}$ to $H^{2} ; T_{\phi} f=P M_{\phi} f$, for $f \in H^{2}(\partial \mathbb{U})$.

(iii) The Hankel operator $H_{\phi}$ is defined as the compression of the "flipped" $M_{\phi}$ onto $H^{2}(\partial \mathbb{U}) ; H_{\phi} f=P J M_{\phi} f$, for $f \in H^{2}(\partial \cup)$, where $J$ is the unitary self-adjoint operator on $L^{2}(\partial \cup)$ (the so-called flip operator) defined by $(J f)(\zeta):=\bar{\zeta} f(\bar{\zeta})$, for $\zeta \in \partial \mathbb{U}$, mapping $H^{2}$ onto $\left(H^{2}\right)^{\perp}$ (the orthogonal complement of $\left.H^{2}\right)$ and $\left(H^{2}\right)^{\perp}$ onto $H^{2}$.

In each case, $\phi$ is called the symbol of the operator.

These classes of operators can also be considered as solutions to some linear operator-equations involving the Toeplitz 
operator $T_{e^{t \theta}}$, known as the unilateral forward shift, and its Hilbert-adjoint $T_{e^{-\theta \theta}}$, usually called the unilateral backward shift. Indeed, it is well known that an operator $H$ is Hankel if and only if $T_{e^{-i \theta}} H=H T_{e^{t \theta}}$ (Hankel equation) and that an operator $T$ is Toeplitz if and only if $T_{e^{-\imath \theta}} T T_{e^{t \theta}}=T$ (Toeplitz equation).

Generalizations of such operator-equations have been studied and explored for some time. For instance, in [9] the operator-equation $S^{*} X T=X$, for arbitrary contractions $S$ and $T$ acting on different Hilbert spaces, has been studied. Pták in [10] studied the solutions to the operator-equation $S^{*} X=X T$, where $S$ and $T$ are contractions.

Here we study an operator-equation, on $\mathscr{B}\left(H^{2}\right)$, which is a slight modification to the Toeplitz equation; namely, $T_{e^{-t \theta}} X T_{e^{i \theta}}=\lambda X$, for an arbitrary complex number $\lambda$. This operator-equation appeared in [11] and it was asked what its operator-solutions could be, what algebraic and operator-theoretic properties those solutions had, and how these operator-solutions relate to the case $\lambda=1$ (Toeplitz operators). Fortunately, this problem is a spectral one; that is, its solutions are the eigen-operators of a bounded operatorvalued linear transformation on $\mathscr{B}\left(H^{2}\right)$, which have been found and characterized by Sun in [12].

In this paper we study and develop some algebraic and operator-theoretic properties of $\lambda$-Toeplitz operators as the bounded operator-solutions to the operator-equation $T_{e^{-t \theta}} X T_{e^{t \theta}}=\lambda X$, for an arbitrary complex number $\lambda$. In most cases, it is shown that $\lambda$-Toeplitz operators behave the same as the classical Toeplitz operators, on $H^{2}$. We also introduce the classes of analytic and coanalytic $\lambda$-Toeplitz operators (Definition 9), which generalize the most commonly considered classes of Toeplitz operators, and apply them to study the multiplicative properties of $\lambda$-Toeplitz operators. In Theorem 14, we show that a product of two $\lambda$-Toeplitz operators is again one precisely when each operator is either analytic or coanalytic, which generalizes [13, Theorem 8]. We then give an example (Example 16) to show that, unlike the Toeplitz case, two analytic (resp., coanalytic) $\lambda$-Toeplitz operators need not commute, (which violates [13, Theorem 9] in the $\lambda$-Toeplitz operators' context). Though, we can still obtain some necessary and sufficient conditions for pairs of (co-)analytic $\lambda$-Toeplitz operators to commute (Theorem 17). We also obtain an interesting result (Corollary 18) on the problem of invertibility for $\lambda$-Toeplitz operators and its connection with our notions of analyticity and coanalyticity, which generalizes [13, Corollary 2]. Finally, we study the relation between analytic/coanalytic $\lambda$-Toeplitz operators and the classical Hankel operators (Theorem 19). This work justifies Barría and Halmos' suggestion, in [11], that the notion of $\lambda$-Toeplitzness may be worthy of study.

Finally, it should be mentioned that this work has its roots in [13] and been inspired by $[12,14]$.

We close this section by setting up the notations required for what is to follow.

\subsection{Notations}

(i) $\mathscr{B}\left(H^{2}\right)$ is the $C^{*}$-algebra of all bounded linear operators on $H^{2}$. (ii) $\mathscr{K}$ stands for the two-sided ideal of all compact operators in $\mathscr{B}\left(H^{2}\right)$.

(iii) For $T \in \mathscr{B}\left(H^{2}\right)$,

$\sigma(T)$ denotes the spectrum of $T$;

$\sigma_{p}(T)$ denotes the set of eigenvalues for $T$.

(iv) The standard tensor notation will be used for operators of rank one: for $f$ and $g$ vectors in $H^{2}$, the operator $f \otimes g$ is defined by $(f \otimes g) h=\langle h, g\rangle_{H^{2}} f$.

(v) The standard orthonormal basis $\left(e_{n}\right)_{n=0}^{+\infty}$ for $H^{2}$, where $e_{n}$ s are functions in $H^{2}$ defined as $e_{n}(\zeta)=\zeta^{n}$, for $\zeta \in$ $\partial \mathbb{U}$.

(vi) For $f \in H^{2}$ and $n=0,1,2, \ldots, \widehat{f}(n)$ stands for the $n$ th-Fourier coefficient of $f$; that is, $\widehat{f}(n)=\left\langle f, e_{n}\right\rangle$.

(vii) $H^{\infty}(\partial \cup)$ consists of all boundary functions of bounded holomorphic functions on $\mathbb{U}$.

\section{2. $\lambda$-Toeplitzness}

Here we give two approaches for defining the concept of " $\lambda$-Toeplitzness": matricial and operator-theoretic approaches.

Definition 1. One calls a singly infinite matrix a $\lambda$-Toeplitz matrix if, on each diagonal (parallel to the main diagonal), the entries are in continued proportion; that is, the matrix $\left(a_{m n}\right)_{m, n=0}^{\infty}$ is a $\lambda$-Toeplitz matrix if there exists a $\lambda \in \mathbb{C}$ such that $a_{m+1, n+1}=\lambda a_{m, n}$, for $m, n=0,1,2, \ldots$

For a fixed complex number $\lambda$, a typical example of a singly infinite $\lambda$-Toeplitz matrix is

$$
\left[\begin{array}{ccccc}
a_{0} & a_{-1} & a_{-2} & a_{-3} & \cdots \\
a_{1} & \lambda a_{0} & \lambda a_{-1} & \lambda a_{-2} & \ddots \\
a_{2} & \lambda a_{1} & \lambda^{2} a_{0} & \lambda^{2} a_{-1} & \ddots \\
a_{3} & \lambda a_{2} & \lambda^{2} a_{1} & \lambda^{3} a_{0} & \ddots \\
\vdots & \ddots & \ddots & \ddots & \ddots
\end{array}\right] .
$$

In [13], it is shown that Toeplitz operators, on $H^{2}$, are the solutions of the operator-equation $T_{e^{-\ell \theta}} X T_{e^{1 \theta}}=X$, on $\mathscr{B}\left(H^{2}\right)$. Equivalently, this means that they are the eigen-operators of the following operator-valued linear transformation (let us call it Toeplitz mapping on $\left.\mathscr{B}\left(H^{2}\right)\right)$ :

$$
\begin{gathered}
\Gamma: \mathscr{B}\left(H^{2}\right) \longrightarrow \mathscr{B}\left(H^{2}\right) \\
X \longmapsto T_{e^{-\imath \theta}} X T_{e^{\imath \theta}},
\end{gathered}
$$

corresponding to the eigenvalue 1 . This suggests a general context in which Toeplitz operators can be embedded.

Definition 2. One calls an operator, in $\mathscr{B}\left(H^{2}\right)$, a $\lambda$-Toeplitz operator if it is an eigen-operator of the Toeplitz mapping $\Gamma$ corresponding to one of its eigenvalues. 
More precisely, for $\lambda \in \sigma_{p}(\Gamma)$, the set $\mathscr{T}_{\lambda}:=\operatorname{ker}(\lambda I-$ $\Gamma)$ consists of $\lambda$-Toeplitz operators corresponding to $\lambda$, or, equivalently,

$$
\mathscr{T}_{\lambda}=\left\{X \in \mathscr{B}\left(H^{2}\right) \mid \Gamma(X)\left(=T_{e^{-\imath \theta}} X T_{e^{\imath \theta}}\right)=\lambda X\right\} .
$$

It can be easily checked that the Toeplitz mapping is a contraction; moreover, since $T_{e^{-\imath \theta}} T_{e^{\imath \theta}}=I$, we should have $\|\Gamma\|=1$. Thus, there is no $\lambda$-Toeplitz operator for $|\lambda|>1$; that is, $\sigma(\Gamma) \subseteq \overline{\mathbb{U}}$. But every diagonal operator with diagonal $\left(\lambda^{n}\right)_{n=0}^{\infty}$, for $\lambda \in \overline{\mathbb{U}}$, is a solution for $[\lambda I-\Gamma](X)=0$. Thus, $\overline{\mathbb{U}} \subseteq \sigma_{p}(\Gamma)(\subseteq \sigma(\Gamma))$. Therefore, $\sigma_{p}(\Gamma)=\sigma(\Gamma)=\overline{\mathbb{U}}$; that is, the only eigen-operators for $\Gamma$ are the ones corresponding to the eigenvalues living in $\overline{\mathbb{U}}$.

Observation 1. Bounded operator-solutions to $T_{e^{-\imath \theta}} X T_{e^{\imath \theta}}=$ $\lambda X$, on $\mathscr{B}\left(H^{2}\right)$, exist if and only if $\lambda \in \overline{\mathbb{U}}$. For more details on the form of a $\lambda$-Toeplitz operator, see [12].

Theorem 3 (Sun [12]). Let $\lambda \in \mathbb{C}$. The operator-equation $T_{e^{-\imath \theta}} X T_{e^{i \theta}}=\lambda X$ has bounded solutions if and only if $\lambda \leq 1$. One then has the following.

(i) If $|\lambda|=1$, all solutions are of the form $D_{\bar{\lambda}} T$, where $T$ is a Toeplitz operator and $D_{\lambda}$ is the diagonal unitary operator defined as $D_{\lambda} e_{n}=\lambda^{n} e_{n}$ for all $n$.

(ii) If $|\lambda|<1$, all solutions are compact operators of the form

$$
\sum_{n=0}^{\infty} \lambda^{n}\left(\left(T_{e^{i n \theta}} f\right) \otimes e_{n}+e_{n} \otimes\left(T_{e^{i n}} g\right)\right)
$$

for some $f$ and $g \in H^{2}$.

For convenience, let us divide $\lambda$-Toeplitz operators into two main classes: unimodular $\lambda$-Toeplitz operators and nonunimodular $\lambda$-Toeplitz operators, which are the ones corresponding to the eigenvalues of the Toeplitz mapping $\Gamma$ on the unit circle and the unit disk, respectively.

Remark 4. Some immediate consequences of Sun's Theorem are that the nonunimodular $\lambda$-Toeplitz operators are compact (so are not invertible) and unimodular $\lambda$-Toeplitz operators are not. Indeed, in the latter case, the only compact unimodular $\lambda$-Toeplitz operator is the zero operator.

Remark 5. By Definition 2, if $X \in \mathscr{T}_{\lambda}$, for some $\lambda \in \overline{\mathbb{U}}$, then $T_{e^{-\imath \theta}} X T_{e^{t \theta}}=\lambda X$. Hence, the entries of its matrix representation $\left(a_{m, n}\right)_{m, n=0}^{\infty}$, with respect to the monomial basis for $H^{2}$, satisfy

$$
\begin{aligned}
a_{m+1, n+1} & =\left\langle X e_{n+1}, e_{m+1}\right\rangle_{H^{2}}=\left\langle X T_{e^{\imath \theta}} e_{n}, T_{e^{\imath \theta}} e_{m}\right\rangle_{H^{2}} \\
& =\left\langle T_{e^{-\imath \theta}} X T_{e^{\imath \theta}} e_{n}, e_{m}\right\rangle_{H^{2}}=\left\langle\lambda X e_{n}, e_{m}\right\rangle_{H^{2}} \\
& =\lambda a_{m, n}, \quad \text { for } m, n=0,1,2, \ldots
\end{aligned}
$$

This yields

$$
\left(a_{m, n}\right)_{m, n=0}^{\infty}=\left[\begin{array}{ccccc}
a_{0,0} & a_{0,1} & a_{0,2} & a_{0,3} & \cdots \\
a_{1,0} & \lambda a_{0,0} & \lambda a_{0,1} & \lambda a_{0,2} & \ddots \\
a_{2,0} & \lambda a_{1,0} & \lambda^{2} a_{0,0} & \lambda^{2} a_{0,1} & \ddots \\
a_{3,0} & \lambda a_{2,0} & \lambda^{2} a_{1,0} & \lambda^{3} a_{0,0} & \ddots \\
\vdots & \ddots & \ddots & \ddots & \ddots
\end{array}\right],
$$

where

$$
X e_{0}=\sum_{n=0}^{\infty} a_{n, 0} e^{i n \theta}, \quad X^{*} e_{0}=\sum_{n=0}^{\infty} \bar{a}_{0, n} e^{\imath n \theta}
$$

\section{Basic Properties of $\lambda$-Toeplitz Operators}

Recall that $\mathscr{T}_{1}$ consists of all (classical) Toeplitz operators, and it turns out, as we will see later, that other $\lambda$-Toeplitz operators behave like them. Also, notice that, for each $\lambda \epsilon$ $\overline{\mathbb{U}}, \mathscr{T}_{\lambda}$ forms a complex vector subspace of $\mathscr{B}\left(H^{2}\right)$.

Here we look at the following straightforward properties of the $\lambda$-Toeplitz operators. First, from Definition 2, we observe that, for each $\lambda \in \overline{\mathbb{U}}, \mathscr{T}_{\lambda}$ is topologically well behaved. Indeed, for $X \in \mathscr{T}_{\lambda}$, since $\Gamma(X)=T_{e^{-\imath \theta}} X T_{e^{\imath \theta}}$ is weakly continuous in its middle factor, $\mathscr{T}_{\lambda}$ is weakly closed, and, therefore, a fortiori, it is strongly and uniformly closed.

The next result, inspired by [14, Theorem 4.5], states that self-adjointness only exists among real $\lambda$-Toeplitz operators; that is, $\lambda$-Toeplitz operators correspond to real eigenvalues for $\Gamma$.

Proposition 6. For $\lambda \in \overline{\mathbb{U}}$ and $X \in \mathscr{T}_{\lambda}$, one has the following.

(i) $X^{*} \in \mathscr{T}_{\bar{\lambda}}$.

(ii) If $X \neq 0$ and $X=X^{*}$, then $\lambda \in \mathbb{R}$.

Proof. (i) Since $X \in \mathscr{T}_{\lambda}$, we have $T_{e^{-\imath \theta}} X T_{e^{t \theta}}=\lambda X$, from which, by taking adjoints, we get $T_{e^{-\imath \theta}} X^{*} T_{e^{\imath \theta}}=\bar{\lambda} X^{*}$. So $X^{*} \in \mathscr{T}_{\bar{\lambda}}$.

(ii) If $X$ is a nonzero self-adjoint element of $\mathscr{T}_{\lambda}$, then $X \in$ $\mathscr{T}_{\lambda} \cap \mathscr{T}_{\bar{\lambda}}$. But this means that

$$
\lambda X=T_{e^{-\imath \theta}} X T_{e^{\imath \theta}}=T_{e^{-\imath \theta}} X^{*} T_{e^{\imath \theta}}=\bar{\lambda} X^{*}=\bar{\lambda} X,
$$

which implies $\lambda=\bar{\lambda}$, or, equivalently, $\lambda \in \mathbb{R}$.

Remark 7. As a consequence of Sun's Theorem, every nonunimodular $\lambda$-Toeplitz operator is compact. This, in turn, states that they are not only noninvertible, but also nonessentially invertible. But the situation is different for unimodular ones. Indeed, the only compact unimodular one is the zero operator: for $\lambda \in \partial \mathbb{U}$, letting $X \in \mathscr{T}_{\lambda}, n$ and $n+k$ be nonnegative integers, we have

$$
\left\langle X e_{n}, e_{n+k}\right\rangle_{H^{2}(\partial \mathbb{U})}=\lambda^{n}\left\langle X e_{0}, e_{k}\right\rangle_{H^{2}(\partial U)} .
$$

Now, if $X$ is a compact operator, then $\left\|X e_{n}\right\|_{H^{2}(\partial \cup)} \rightarrow 0$, as $n \rightarrow \infty$; it follows that $\left\langle X e_{0}, e_{k}\right\rangle_{H^{2}(\partial \cup)}=0$, for all nonnegative $k$.

And, if we apply the same procedure for $X^{*}$, we obtain $\left\langle X^{*} e_{0}, e_{k}\right\rangle_{H^{2}(\partial \mathrm{U})}=0$, for all nonnegative $k$. Therefore, $X=0$. 


\section{Analyticity and Coanalyticity of $\lambda$-Toeplitz Operators}

In [13, p. 96], analyticity and coanalyticity of a (classical) Toeplitz operator are defined and characterized in terms of its commutativity with $T_{e^{\imath \theta}}$ and $T_{e^{-\imath \theta}}$, respectively [13, Theorem 7]. Here, we define and give an analogous characterization of these two properties in the $\lambda$-Toeplitz operators' setting. But let us first assign them a "symbol" (similar to one in the classical case) as a generating function.

Definition 8. For $\lambda \in \overline{\mathbb{U}}$, let $X \in \mathscr{T}_{\lambda}$. The symbol of $X$ is defined to be

$$
X e_{0}+\overline{X^{*} e_{0}}-\left\langle X e_{0}, e_{0}\right\rangle_{H^{2}}
$$

and is denoted by $\operatorname{sym}(X)$, in which $X e_{0}$ is called the analytic symbol and $\overline{X^{*} e_{0}}$ is called the coanalytic symbol of $X$.

Observation 2. For a $\lambda$-Toeplitz operator $X, \operatorname{sym}(X)$ is the function whose nonnegative Fourier coefficients are the terms of the 0th-column of its matrix representation, with respect to the monomial basis for $\mathrm{H}^{2}$, and whose nonpositive Fourier coefficients are the terms of the 0th-row of that matrix.

Definition 8 determines two $H^{2}$-functions, namely, $X e_{0}$ and $X^{*} e_{0}$, by which we may characterize the properties of analyticity and coanalyticity, for such operators, as follows.

Definition 9. A $\lambda$-Toeplitz operator, $X$, is called

(i) analytic if $\operatorname{sym}(X)$ is an analytic function (i.e., $X^{*} e_{0}$ is the constant function $\left.\left\langle e_{0}, X e_{0}\right\rangle_{H^{2}}\right)$;

(ii) coanalytic if $\operatorname{sym}(X)$ is a coanalytic function (i.e., $X e_{0}$ is the constant function $\left.\left\langle X e_{0}, e_{0}\right\rangle_{H^{2}}\right)$.

This definition makes the following remark obvious.

Remark 10. For $\lambda \in \overline{\mathbb{U}}$, letting $X \in \mathscr{T}_{\lambda}$

(i) $X$ is analytic if and only if $\left\langle X^{*} e_{0}, e_{n}\right\rangle_{H^{2}}=0$, for all $n>0$ (i.e., $T_{e^{-\imath \theta}} X^{*} e_{0}=0$ ),

(ii) $X$ is coanalytic if and only if $\left\langle X e_{0}, e_{n}\right\rangle_{H^{2}}=0$, for all $n>0$ (i.e., $T_{e^{-\imath \theta}} X e_{0}=0$ ).

Hence, $X$ is analytic if and only if $X^{*}$ is coanalytic.

Observation 3. Notice that for, $\lambda \in \overline{\mathbb{U}}$ and $\phi \in L^{\infty}(\partial \mathbb{U})$, $D_{\lambda} T_{\phi} \in \mathscr{T}_{\lambda}$, where $D_{\lambda}$ is the diagonal operator with diagonal $\left(\lambda^{n}\right)_{n=0}^{\infty}$. Indeed,

$$
\begin{aligned}
T_{e^{-\imath \theta}} D_{\lambda} T_{\phi} T_{e^{\imath \theta}}= & T_{e^{-\imath \theta}} D_{\lambda}\left(T_{e^{\imath \theta}} T_{e^{-\imath \theta}}+e_{0} \otimes e_{0}\right) T_{\phi} T_{e^{\imath \theta}} \\
= & \left(T_{e^{-\imath \theta}} D_{\lambda} T_{e^{\imath \theta}}\right)\left(T_{e^{-\imath \theta}} T_{\phi} T_{e^{\imath \theta}}\right) \\
& +T_{e^{-\imath \theta}} D_{\lambda} e_{0} \otimes T_{e^{-\imath \theta} \bar{\phi}} e_{0} \\
= & \lambda D_{\lambda} T_{\phi}+\underbrace{T_{e^{-\imath \theta} e_{0}}}_{=0} \otimes T_{e^{-\imath \theta} \bar{\phi}} e_{0} \\
= & \lambda D_{\lambda} T_{\phi} .
\end{aligned}
$$

This observation provides us with two classes of typical examples of analytic and coanalytic $\lambda$-Toeplitz operators.

(1) For $\lambda \in \overline{\mathbb{U}}$ and $\phi \in H^{\infty}(\partial \mathbb{U}), D_{\lambda} T_{\phi}$ is an analytic $\lambda$-Toeplitz operator. Indeed for $n=1,2, \ldots$,

$$
\begin{aligned}
\left\langle\left(D_{\lambda} T_{\phi}\right)^{*} e_{0}, e_{n}\right\rangle_{H^{2}} & =\left\langle D_{\bar{\lambda}} e_{0}, \phi e_{n}\right\rangle \\
& =\left\langle e_{0}, \phi e_{n}\right\rangle=\hat{\bar{\phi}}(n)=0 .
\end{aligned}
$$

(2) For $\lambda \in \overline{\mathbb{U}}$ and $\bar{\phi} \in H^{\infty}(\partial \mathbb{U}), D_{\lambda} T_{\phi}$ is a coanalytic $\lambda$-Toeplitz operator. Indeed for $n=1,2, \ldots$,

$$
\begin{aligned}
\left\langle D_{\lambda} T_{\phi} e_{0}, e_{n}\right\rangle_{H^{2}} & =\left\langle T_{\phi} e_{0}, D_{\bar{\lambda}} e_{n}\right\rangle \\
& =\left\langle T_{\phi} e_{0}, \bar{\lambda}^{n} e_{n}\right\rangle=\lambda^{n} \widehat{\phi}(n)=0 .
\end{aligned}
$$

Note that if $X \in \mathscr{T}_{\lambda}$ is analytic, its matrix representation with respect to the monomial basis for $H^{2}$ is lower triangular. Indeed, for $m, n=0,1,2, \ldots$ with $m<n$

$$
\begin{aligned}
\left\langle X e_{n}, e_{m}\right\rangle_{H^{2}} & =\left\langle X T_{e^{\imath \theta}}^{m} e_{n-m}, T_{e^{\imath \theta}}^{m} e_{0}\right\rangle \\
& =\left\langle T_{e^{-\imath \theta}}^{m} X T_{e^{\imath \theta}}^{m} e_{n-m}, e_{0}\right\rangle=\left\langle\lambda^{m} X e_{n-m}, e_{0}\right\rangle \\
& =\lambda^{m}\left\langle e_{n-m}, X^{*} e_{0}\right\rangle=0 .
\end{aligned}
$$

With the same reasoning one can show that coanalytic $\lambda$-Toeplitz operators correspond to upper triangular $\lambda$-Toeplitz matrices.

Before stating the first result of this section, we need to introduce some terms. For a Hilbert space bounded operator $A$, consider the operator-equation

$$
A X=\lambda X A
$$

for some complex number $\lambda$. If there is a nonzero (bounded) operator $X$ and a scalar $\lambda$ as above that satisfy (16), according to [15], it is said that $A \lambda$-commutes with $X$ and that $\lambda$ is an extended eigenvalue and $X$ is an extended eigen-operator of $A$.

Equation (16) has been studied in [16] and, independently, in [17]. These works provided extensions of Lomonosov's classic result [18].

Now, we use these terms to state our next result which characterizes analyticity and coanalyticity of $\lambda$-Toeplitz operators in terms of their $\lambda$-commutativity with $T_{e^{\imath \theta}}$ and $T_{e^{-\imath \theta}}$.

Theorem 11. Let $\lambda \in \overline{\mathbb{U}} \backslash\{0\}$ and $X \in \mathscr{B}\left(H^{2}\right)$. A necessary and sufficient condition that $X$ is an analytic (coanalytic) $\lambda$-Toeplitz operator in $\mathscr{T}_{\lambda}$ is that it $\lambda$ commutes with $T_{e^{\imath \theta}}\left(\lambda^{-1}\right.$-commutes with $\left.T_{e^{-\imath \theta}}\right)$; that is, $X T_{e^{i \theta}}=$ $\lambda T_{e^{\imath \theta}} X\left(T_{e^{-\imath \theta}} X=\lambda X T_{e^{-\imath \theta}}\right)$.

Proof. (i) Let $X$ be an analytic $\lambda$-Toeplitz operator in $\mathscr{T}_{\lambda}$; that is, $T_{e^{-\imath \theta}} X^{*} e_{0}=0$. Hence,

$$
\begin{aligned}
\lambda T_{e^{\imath \theta}} X & =T_{e^{\imath \theta}}(\lambda X)=T_{e^{\imath \theta}}\left(T_{e^{-\imath \theta}} X T_{e^{\imath \theta}}\right) \\
& =\left(I-e_{0} \otimes e_{0}\right) X T_{e^{\imath \theta}} \\
& =X T_{e^{\imath \theta}}-\left(e_{0} \otimes T_{e^{-i \theta}} X^{*} e_{0}\right)=X T_{e^{\imath \theta}} .
\end{aligned}
$$


Now, let $X \in \mathscr{B}\left(H^{2}\right)$ be such that $X T_{e^{i \theta}}=\lambda T_{e^{\imath \theta}} X$. Just by multiplying both sides by $T_{e^{-\imath \theta}}$ from the left, one can easily see that $X \in \mathscr{T}_{\lambda}$. To show it is analytic, we need to prove $\left\langle X^{*} e_{0}, e_{n}\right\rangle=0$, for $n=1,2, \ldots$. So,

$$
\begin{aligned}
\left\langle X^{*} e_{0}, e_{n}\right\rangle_{H^{2}} & =\left\langle e_{0}, X T_{e^{\imath \theta} e_{n-1}}\right\rangle_{H^{2}}=\left\langle e_{0}, \lambda T_{e^{\imath \theta}} X e_{n-1}\right\rangle_{H^{2}} \\
& =\bar{\lambda}\left\langle X^{*} T_{e^{-\imath \theta} e_{0}}, e_{n-1}\right\rangle_{H^{2}}=0,
\end{aligned}
$$

which means $X$ is an analytic $\lambda$-Toeplitz operator.

(ii) If $X$ is a coanalytic $\lambda$-Toeplitz operator in $\mathscr{T}_{\lambda}$, that is, $T_{e^{-i \theta}} X e_{0}=0$, then

$$
\begin{aligned}
\lambda X T_{e^{-\imath \theta}} & =\left(T_{e^{-\imath \theta}} X T_{e^{\imath \theta}}\right) T_{e^{-\imath \theta}}=T_{e^{-\imath \theta}} X\left(I-e_{0} \otimes e_{0}\right) \\
& =T_{e^{-\imath \theta}} X-\left(T_{e^{-\imath \theta}} X e_{0} \otimes e_{0}\right)=T_{e^{-\imath \theta}} X .
\end{aligned}
$$

Now, let $X \in \mathscr{B}\left(H^{2}\right) \lambda^{-1}$-commute with $T_{e^{-\imath \theta}}$; that is, $T_{e^{-\imath \theta}} X=$ $\lambda X T_{e^{-\imath \theta}}$. Multiplying both sides by $T_{e^{\imath t}}$ from the right shows that $X \in \mathscr{T}_{\lambda}$. To prove coanalyticity, we need to show $T_{e^{-\imath \theta}} X e_{0}=0$. So, we have

$$
\left(T_{e^{-\imath \theta}} X\right) e_{0}=\left(\lambda X T_{e^{-\imath \theta}}\right) e_{0}=0 .
$$

Remark 12. Using the terms aforementioned, Theorem 11 can also be restated as follows.

\section{Theorem $11^{\prime}$}

(i) Let $\lambda \in \overline{\mathbb{U}} \backslash\{0\}$. A necessary and sufficient condition in which $X \in \mathscr{T}_{\lambda}$ is analytic is that $T_{e^{i \theta}}$ is an extended eigen-operator of $A$ corresponding to the extended eigenvalue $\lambda$.

(ii) Let $\lambda \in \overline{\mathbb{U}} \backslash\{0\}$. A necessary and sufficient condition in which $X \in \mathscr{T}_{\lambda}$ is coanalytic is that $T_{e^{-t \theta}}$ is an extended eigen-operator of $A$ corresponding to the extended eigenvalue $\lambda^{-1}$.

Remark 13. Notice that if $X \in \mathscr{T}_{0}$, it can be represented by the finite-rank operator $\left[\left(X e_{0}-\left\langle X e_{0}, e_{0}\right\rangle_{H^{2}} e_{0}\right) \otimes e_{0}\right]+e_{0} \otimes X^{*} e_{0}$. Thus,

(1) $X$ is analytic if and only if $X^{*} e_{0}$ is the constant function $\left\langle e_{0}, X e_{0}\right\rangle_{H^{2}}$ and, in this case, $X=X e_{0} \otimes e_{0}$.

(2) And $X$ is coanalytic if and only if $X e_{0}$ is the constant function $\left\langle X e_{0}, e_{0}\right\rangle_{H^{2}}$ and, in this case, $X=e_{0} \otimes X^{*} e_{0}$.

\section{Multiplicative Properties of $\lambda$-Toeplitz Operators}

Although, for a fixed $\lambda \in \overline{\mathbb{U}}, \mathscr{T}_{\lambda}$ is closed under finite summation of its elements, the corresponding result rarely holds for products. As an application of Theorem 11, we will see that $\lambda$-Toeplitzness is preserved under multiplication, on the right, by analytic $\lambda$-Toeplitz operators and, on the left, by coanalytic ones.

Theorem 14. For $\lambda_{1}, \lambda_{2} \in \overline{\mathbb{U}}$, let $X_{1} \in \mathscr{T}_{\lambda_{1}}$ and $X_{2} \in \mathscr{T}_{\lambda_{2}}$.
A necessary and sufficient condition that the product $X_{1} X_{2}$ is a $\lambda$-Toeplitz operator in $\mathscr{T}_{\lambda_{1} \lambda_{2}}$ is that either $X_{1}$ is coanalytic or $X_{2}$ is analytic.

Proof. Let us first assume $X_{1} X_{2} \in \mathscr{T}_{\lambda_{1} \lambda_{2}}$. Hence,

$$
\begin{aligned}
\lambda_{1} \lambda_{2} X_{1} X_{2}= & T_{e^{-\imath \theta}} X_{1} X_{2} T_{e^{\imath \theta}} \\
= & T_{e^{-\imath \theta}} X_{1}\left(T_{e^{\imath \theta}} T_{e^{-\imath \theta}}+e_{0} \otimes e_{0}\right) X_{2} T_{e^{\imath \theta}} \\
= & \left(T_{e^{-\imath \theta}} X_{1} T_{e^{\imath \theta}}\right)\left(T_{e^{-\imath \theta}} X_{2} T_{e^{\imath \theta}}\right) \\
& +\left(T_{e^{-\imath \theta}} X_{1} e_{0} \otimes T_{e^{-\imath \theta}} X_{2}^{*} e_{0}\right) \\
= & \lambda_{1} \lambda_{2} X_{1} X_{2}+\left(T_{e^{-\imath \theta}} X_{1} e_{0} \otimes T_{e^{-\imath \theta}} X_{2}^{*} e_{0}\right) .
\end{aligned}
$$

So, for holding the equality, we should have either $T_{e^{-\imath \theta}} X_{1} e_{0}=$ 0 or $T_{e^{-\imath \theta}} X_{2}^{*} e_{0}=0$; that is, either $X_{1}$ is coanalytic or $X_{2}$ is analytic.

Let us suppose, for now, $X_{1}$ is a coanalytic $\lambda$-Toeplitz operator in $\mathscr{T}_{\lambda_{1}}$ and $X_{2} \in \mathscr{T}_{\lambda_{2}}$. To show that $X_{1} X_{2} \in \mathscr{T}_{\lambda_{1} \lambda_{2}}$, we apply Theorem 11 to write

$$
\begin{aligned}
T_{e^{-\imath \theta}} X_{1} X_{2} T_{e^{\imath \theta}} & =\lambda_{1} X_{1} T_{e^{-\imath \theta}} X_{2} T_{e^{\imath \theta}} \\
& =\left(\lambda_{1} X_{1}\right)\left(\lambda_{2} X_{2}\right)=\lambda_{1} \lambda_{2} X_{1} X_{2},
\end{aligned}
$$

which proves $X_{1} X_{2}$ is a $\lambda$-Toeplitz operator in $\mathscr{T}_{\lambda_{1} \lambda_{2}}$.

And if $X_{2}$ is an analytic $\lambda$-Toeplitz operator in $\mathscr{T}_{\lambda_{2}}$ and $X_{1} \in \mathscr{T}_{\lambda_{1}}$, again using Theorem 11 results in

$$
\begin{aligned}
T_{e^{-\imath \theta}} X_{1} X_{2} T_{e^{\imath \theta}} & =T_{e^{-\imath \theta}} X_{1}\left(\lambda_{2} T_{e^{\imath \theta}} X_{2}\right) \\
& =\lambda_{2}\left(T_{e^{-\imath \theta}} X_{1} T_{e^{\imath \theta}}\right) X_{2}=\lambda_{1} \lambda_{2} X_{1} X_{2},
\end{aligned}
$$

which proves the same thing.

From the proof of Theorem 14, along with considering Remark 7, one may deduce the following property for unimodular $\lambda$-Toeplitz operators.

Proposition 15. For $\lambda_{1}, \lambda_{2} \in \partial \mathbb{U}$, let $X_{1} \in \mathscr{T}_{\lambda_{1}}$ and $X_{2} \in$ $\mathscr{T}_{\lambda_{2}}$. If $X_{1} X_{2} \in \mathscr{T}_{\mu} \backslash\{0\}$ for some $\mu \in \partial \mathbb{U}$, then $\mu=\lambda_{1} \lambda_{2}$. Moreover, either $X_{1}$ is coanalytic or $X_{2}$ is analytic.

Proof. Considering the assumptions, we have

$$
\begin{aligned}
\mu X_{1} X_{2}= & T_{e^{-\imath \theta}} X_{1} X_{2} T_{e^{\imath \theta}} \\
= & T_{e^{-\imath \theta}} X_{1}\left(T_{e^{\imath \theta}} T_{e^{-\imath \theta}}+e_{0} \otimes e_{0}\right) X_{2} T_{e^{\imath \theta}} \\
= & \left(T_{e^{-\imath \theta}} X_{1} T_{e^{\imath \theta}}\right)\left(T_{e^{-\imath \theta}} X_{2} T_{e^{\imath \theta}}\right) \\
& +\left(T_{e^{-\imath \theta}} X_{1} e_{0} \otimes T_{e^{-\imath \theta}} X_{2}^{*} e_{0}\right) \\
= & \lambda_{1} \lambda_{2} X_{1} X_{2}+\left(T_{e^{-\imath \theta}} X_{1} e_{0} \otimes T_{e^{-\imath \theta}} X_{2}^{*} e_{0}\right),
\end{aligned}
$$

which implies

$$
\left(\mu-\lambda_{1} \lambda_{2}\right) X_{1} X_{2}=T_{e^{-\imath \theta}} X_{1} e_{0} \otimes T_{e^{-\imath \theta}} X_{2}^{*} e_{0} .
$$

Since $\mu \neq 0$ and $X_{1} X_{2}$ is a nonzero $\lambda$-Toeplitz operator in $\mathscr{T}_{\mu}, X_{1} X_{2}$ cannot be of finite rank (see Remark 7). Hence, both sides in (25) should be zero. Therefore, $\mu=\lambda_{1} \lambda_{2}$, and this in turn implies that either $X_{1}$ should be coanalytic or $X_{2}$ is analytic. 
Recall that if the symbols of two (classical) Toeplitz operators are either analytic or coanalytic, they necessarily commute [13, Theorem 9]. But, surprisingly, this is not the case among $\lambda$-Toeplitz operators; for, let us look at an example.

Example 16. For some $\lambda \in \overline{\mathbb{U}} \backslash\{1\}$,

(1) let $X_{1}, X_{2} \in \mathscr{T}_{\lambda}$ be analytic such that $X_{2}$ is arbitrary and $X_{1}$ is given by

$$
\left\langle X_{1} e_{j}, e_{i}\right\rangle_{H^{2}}=\lambda^{j} \delta_{i, j+1} \quad i, j=0,1,2, \ldots,
$$

where $\delta_{i, j+1}$ is the Kronecker delta. Note that $X_{2}$ can also be represented as

$$
\left\langle X_{2} e_{j}, e_{i}\right\rangle_{H^{2}}= \begin{cases}0 & \text { if } i<j \\ \lambda^{j}\left\langle X_{2} e_{0}, e_{i-j}\right\rangle_{H^{2}} & \text { if } i \geq j .\end{cases}
$$

Hence, we have

$$
\begin{aligned}
\left\langle X_{1} X_{2} e_{j}, e_{i}\right\rangle_{H^{2}} & = \begin{cases}0 & \text { if } i \leq j \\
\lambda^{i+j-1}\left\langle X_{2} e_{0}, e_{i-j-1}\right\rangle_{H^{2}} & \text { if } i>j,\end{cases} \\
\left\langle X_{2} X_{1} e_{j}, e_{i}\right\rangle_{H^{2}} & = \begin{cases}0 & \text { if } i \leq j \\
\lambda^{2 j+1}\left\langle X_{2} e_{0}, e_{i-j-1}\right\rangle_{H^{2}} & \text { if } i>j .\end{cases}
\end{aligned}
$$

Therefore, as analytic $\lambda$-Toeplitz operators, $X_{1}$ and $X_{2}$ do not commute.

(2) In the other direction, consider two coanalytic $\lambda$-Toeplitz operators $X_{3}, X_{4} \in \mathscr{T}_{\lambda}$, such that $X_{4}$ is arbitrary and $X_{3}=X_{1}^{*}$, the Hilbert space adjoint of $X_{1}$ in the previous case; that is,

$$
\left\langle X_{3} e_{j}, e_{i}\right\rangle_{H^{2}}=\bar{\lambda}^{i} \delta_{j, i+1} \quad i, j=0,1,2, \ldots
$$

Again, note that $X_{4}$ can also be represented as

$$
\left\langle X_{4} e_{j}, e_{i}\right\rangle_{H^{2}}= \begin{cases}0 & \text { if } i>j \\ \lambda^{i}\left\langle X_{4} e_{j-i}, e_{0}\right\rangle_{H^{2}} & \text { if } i \leq j .\end{cases}
$$

Hence, we have

$$
\begin{aligned}
\left\langle X_{3} X_{4} e_{j}, e_{i}\right\rangle_{H^{2}} & = \begin{cases}0 & \text { if } j \leq i \\
|\lambda|^{2 i} \lambda\left\langle X_{4} e_{j-i-1}, e_{0}\right\rangle_{H^{2}} & \text { if } j>i,\end{cases} \\
\left\langle X_{4} X_{3} e_{j}, e_{i}\right\rangle_{H^{2}} & = \begin{cases}0 & \text { if } j \leq i \\
\bar{\lambda}^{j-1} \lambda^{i}\left\langle X_{4} e_{j-i-1}, e_{0}\right\rangle_{H^{2}} & \text { if } j>i .\end{cases}
\end{aligned}
$$

Therefore, as coanalytic $\lambda$-Toeplitz operators, $X_{3}$ and $X_{4}$ do not commute.

Though, we can still obtain some necessary and sufficient conditions for pairs of (co-)analytic $\lambda$-Toeplitz operators to commute.
Theorem 17. For $\lambda_{1}, \lambda_{2} \in \overline{\mathbb{U}}$, let $X_{1} \in \mathscr{T}_{\lambda_{1}}$ and $X_{2} \in \mathscr{T}_{\lambda_{2}}$.

(i) If both $X_{1}$ and $X_{2}$ are analytic, then $X_{1} X_{2}=$ $X_{2} X_{1}$ if and only if $\left(X_{2} e_{0}\right)\left(\lambda_{1} \zeta\right)\left(X_{1} e_{0}\right)(\zeta)=\left(X_{1} e_{0}\right)$ $\left(\lambda_{2} \zeta\right)\left(X_{2} e_{0}\right)(\zeta)$, for almost all $\zeta \in \partial \mathbb{U}$.

(ii) If both $X_{1}$ and $X_{2}$ are coanalytic, then $X_{1} X_{2}=$ $X_{2} X_{1}$ if and only if $\left(X_{2}^{*} e_{0}\right)\left(\overline{\lambda_{1}} \zeta\right)\left(X_{1}^{*} e_{0}\right)(\zeta)=\left(X_{1}^{*} e_{0}\right)$ $\left(\overline{\lambda_{2}} \zeta\right)\left(X_{2}^{*} e_{0}\right)(\zeta)$, for almost all $\zeta \in \partial \mathbb{U}$.

Proof. (i) Assume that $X_{1} \in \mathscr{T}_{\lambda_{1}}$ and $X_{2} \in \mathscr{T}_{\lambda_{2}}$ are both analytic $\lambda$-Toeplitz operators such that $X_{2} e_{0}\left(\lambda_{1} \zeta\right) X_{1} e_{0}(\zeta)=$ $X_{1} e_{0}\left(\lambda_{2} \zeta\right) X_{2} e_{0}(\zeta)$, for almost all $\zeta \in \partial \mathbb{U}$. So, by Theorem 14, $X_{1} X_{2}, X_{2} X_{1} \in \mathscr{T}_{\lambda_{1} \lambda_{2}}$. Also, analyticity of $X_{1}$ and $X_{2}$ reveals that

$$
\begin{aligned}
\left\langle X_{1} e_{j}, e_{i}\right\rangle_{H^{2}} & = \begin{cases}0 & i<j \\
\lambda_{1}^{j}\left\langle X_{1} e_{0}, e_{i-j}\right\rangle_{H^{2}} & i \geq j,\end{cases} \\
\left\langle X_{2} e_{j}, e_{i}\right\rangle_{H^{2}} & = \begin{cases}0 & i<j \\
\lambda_{2}^{j}\left\langle X_{2} e_{0}, e_{i-j}\right\rangle_{H^{2}} & i \geq j,\end{cases}
\end{aligned}
$$

which in turn implies

$$
\left\langle X_{1} X_{2} e_{j}, e_{i}\right\rangle= \begin{cases}0 & i<j \\ \sum_{k=j}^{i} \lambda_{1}^{k} \lambda_{2}^{j}\left\langle X_{1} e_{0}, e_{i-k}\right\rangle\left\langle X_{2} e_{0}, e_{k-j}\right\rangle & i \geq j .\end{cases}
$$

But since $X_{1} X_{2}$ is an analytic $\lambda$-Toeplitz operator, we just need to consider the Fourier coefficients of $X_{1} X_{2} e_{0}$, which can be obtained from the finite sum in (33) by letting $j=0$, which gives us the $i$ th-Fourier coefficient of $X_{1} X_{2} e_{0}$; that is,

$$
\sum_{k=0}^{i} \lambda_{1}^{k}\left\langle X_{2} e_{0}, e_{k}\right\rangle_{H^{2}}\left\langle X_{1} e_{0}, e_{i-k}\right\rangle_{H^{2}}
$$

which is nothing but the $i$ th-Fourier coefficient of $\left(X_{2} e_{0}\right)$ $\left(\lambda_{1} \zeta\right)\left(X_{1} e_{0}\right)(\zeta)$, for almost all $\zeta \in \partial \mathbb{U}$, since

$$
\begin{aligned}
& \left(X_{2} e_{0}\right)\left(\lambda_{1} \zeta\right)\left(X_{1} e_{0}\right)(\zeta) \\
& =\left(\sum_{i=0}^{\infty} \widehat{X_{2} e_{0}}(i) \lambda_{1}^{i} \zeta^{i}\right)\left(\sum_{i=0}^{\infty} \widehat{X_{1} e_{0}}(i) \zeta^{i}\right) \\
& =\sum_{i=0}^{\infty}\left(\sum_{k=0}^{i} \lambda_{1}^{k} \widehat{X_{2} e_{0}}(k) \widehat{X_{1} e_{0}}(i-k)\right) \zeta^{i},
\end{aligned}
$$

where $\widehat{X_{j} e_{0}}(i)=\left\langle X_{j} e_{0}, e_{i}\right\rangle_{H^{2}}$, for $j=1,2$ and $i=0,1,2, \ldots$.

By the assumption $\left(X_{2} e_{0}\right)\left(\lambda_{1} \zeta\right)\left(X_{1} e_{0}\right)(\zeta)=\left(X_{1} e_{0}\right)\left(\lambda_{2} \zeta\right)$ $\left(X_{2} e_{0}\right)(\zeta)$, hence the finite sum in (34) is also equal to the $i$ thFourier coefficient of $\left(X_{1} e_{0}\right)\left(\lambda_{2} \zeta\right)\left(X_{2} e_{0}\right)(\zeta)$; that is,

$$
\sum_{k=0}^{i} \lambda_{2}^{k}\left\langle X_{1} e_{0}, e_{k}\right\rangle_{H^{2}}\left\langle X_{2} e_{0}, e_{i-k}\right\rangle_{H^{2}},
$$

which is the $i$ th-Fourier coefficient of $X_{2} X_{1} e_{0}$. What we already showed is that the $i$ th-Fourier coefficients of 
$\operatorname{sym}\left(X_{1} X_{2}\right)$ and $\operatorname{sym}\left(X_{2} X_{1}\right)$ are equal. Therefore, $X_{1} X_{2}=$ $X_{2} X_{1}$. This proves the sufficiency condition in (37).

Let us assume that $X_{1} X_{2}=X_{2} X_{1}$. This assumption, along with analyticity of $X_{1}$ and $X_{2}$, implies

$$
\begin{aligned}
& \sum_{k=j}^{i} \lambda_{1}^{k} \lambda_{2}^{j}\left\langle X_{1} e_{0}, e_{i-k}\right\rangle\left\langle X_{2} e_{0}, e_{k-j}\right\rangle \\
& =\sum_{k=j}^{i} \lambda_{2}^{k} \lambda_{1}^{j}\left\langle X_{2} e_{0}, e_{i-k}\right\rangle\left\langle X_{2} e_{0}, e_{k-j}\right\rangle
\end{aligned}
$$

for $i, j=0,1,2, \ldots$, such that $i \geq j$. Now, letting $j=0$ in (37), we obtain

$$
\left(X_{2} e_{0}\right)\left(\lambda_{1} \zeta\right)\left(X_{1} e_{0}\right)(\zeta)=\left(X_{1} e_{0}\right)\left(\lambda_{2} \zeta\right)\left(X_{2} e_{0}\right)(\zeta)
$$

for almost all $\zeta \in \partial \mathbb{U}$. This proves the necessity condition in (37).

(ii) Assume that $X_{1} \in \mathscr{T}_{\lambda_{1}}$ and $X_{2} \in \mathscr{T}_{\lambda_{2}}$ are both coanalytic $\lambda$-Toeplitz operators such that

$$
\left(X_{2}^{*} e_{0}\right)\left(\overline{\lambda_{1}} \zeta\right)\left(X_{1}^{*} e_{0}\right)(\zeta)=\left(X_{1}^{*} e_{0}\right)\left(\overline{\lambda_{2}} \zeta\right)\left(X_{2}^{*} e_{0}\right)(\zeta)
$$

for almost all $\zeta \in \partial \mathbb{U}$. Their coanalyticity implies that $X_{1}^{*} \in$ $\mathscr{T} \overline{\lambda_{1}}$ and $X_{2}^{*} \in \mathscr{T} \overline{\lambda_{2}}$ are analytic, which satisfy (39). Therefore, by the sufficiency condition in (37), they commute. This in turn implies that $X_{1}$ and $X_{2}$ commute. This proves the necessity condition in (37).

Now, suppose coanalytic $\lambda$-Toeplitz operators $X_{1}$ and $X_{2}$ commute, which means analytic $\lambda$-Toeplitz operators $X_{1}^{*} \epsilon$ $\mathscr{T} \overline{\lambda_{1}}$ and $X_{2}^{*} \in \mathscr{T}_{\overline{\lambda_{2}}}$ commute. Hence, by the necessity condition in (37), we should have

$$
\left(X_{2}^{*} e_{0}\right)\left(\overline{\lambda_{1}} \zeta\right)\left(X_{1}^{*} e_{0}\right)(\zeta)=\left(X_{1}^{*} e_{0}\right)\left(\overline{\lambda_{2}} \zeta\right)\left(X_{2}^{*} e_{0}\right)(\zeta)
$$

which proves the sufficiency condition in (37).

Another consequence of Theorem 11 characterizes the $\lambda$-Toeplitz operators having $\lambda$-Toeplitz operator inverses.

Corollary 18. For $\lambda \in \partial \mathbb{U}$, let $X \in \mathscr{T}_{\lambda}$. If $X$ is invertible, then a necessary and sufficient condition that $X^{-1}$ is a $\lambda$-Toeplitz operator is that $X$ is either analytic or coanalytic.

Proof. Suppose that $X$ is invertible. If $X$ is analytic, then, by Theorem 11, $X \lambda$-commutes with $T_{e^{t \theta}}$; that is,

$$
X T_{e^{\imath \theta}}=\lambda T_{e^{\imath \theta}} X
$$

from which follows

$$
X^{-1} T_{e^{\imath \theta}}=\bar{\lambda} T_{e^{\imath \theta}} X^{-1}
$$

But, on one hand, (42) implies that $X^{-1}$ is a $\lambda$-Toeplitz operator in $\mathscr{T}_{\bar{\lambda}}$ and, on the other hand, that it is an analytic $\lambda$-Toeplitz operator, using Theorem 11.

The case for coanalyticity walks through the same steps as the latter case.
Suppose now that $X^{-1}$ is known to be a $\lambda$-Toeplitz operator in $\mathscr{T}_{\mu}$, for some $\mu \in \partial \mathbb{U}$. Having the following operator-equations,

$$
\begin{aligned}
T_{e^{-\imath \theta}} X T_{e^{\imath \theta}} & =\lambda X, \\
T_{e^{-\imath \theta}} X^{-1} T_{e^{\imath \theta}} & =\mu X^{-1},
\end{aligned}
$$

we obtain

$$
\begin{gathered}
T_{e^{-\imath \theta}} X e_{0} \otimes T_{e^{-\imath \theta}}\left(X^{-1}\right)^{*} e_{0}=(1-\lambda \mu) I, \\
T_{e^{-\imath \theta}} X^{-1} e_{0} \otimes T_{e^{-\imath \theta}} X^{*} e_{0}=(1-\lambda \mu) I,
\end{gathered}
$$

each of which implies $\mu=\bar{\lambda}$; that is, $X^{-1} \in \mathscr{T}_{\bar{\lambda}}$, and, in this case, from the first equation follows either $X$ is coanalytic or $X^{-1}$ is analytic. And the second one also implies either $X^{-1}$ is coanalytic or $X$ is analytic.

If $X$ is not coanalytic, then $X^{-1}$ is analytic and not constant; this implies that $X^{-1}$ is not coanalytic and hence that $X$ is analytic. The same reasoning also works when it is assumed that $X$ is not analytic.

\section{6. $\lambda$-Toeplitzness versus Hankelness}

One of the properties of Hankelness is that it is preserved under multiplication, on the right, by analytic Toeplitz operators or, on the left, by coanalytic Toeplitz operators. Indeed, if $H, T \in \mathscr{B}\left(H^{2}\right)$ such that $H$ is a Hankel operator and $T$ is an analytic Toeplitz operator, then

$$
T_{e^{-\imath \theta}} H T=H T_{e^{\imath \theta}} T=H T T_{e^{t \theta}},
$$

which states that HT satisfies the Hankel equation, so is a Hankel operator. A similar way shows that TH is a Hankel operator, where $T$ is a coanalytic Toeplitz operator.

It turns out that Hankel operators behave in a similar manner when they meet analytic/coanalytic $\lambda$-Toeplitz operators.

Theorem 19. Let $H \in \mathscr{B}\left(H^{2}\right)$ be a Hankel operator and $\lambda \in \overline{\mathbb{U}}$. If $X_{1}$ is an analytic and $X_{2}$ is a coanalytic $\lambda$-Toeplitz operator in $\mathscr{T}_{\lambda}$, then $H X_{1}$ and $X_{2} H$ satisfy the Hankel equation in the sense that $\lambda T_{e^{-\imath \theta}} H X_{1}=H X_{1} T_{e^{t \theta}}$ and $T_{e^{-\imath \theta}} X_{2} H=\lambda X H T_{e^{i \theta}}$.

Proof. As it is well known, $H$ is a Hankel operator if and only if $T_{e^{-\imath \theta}} H=H T_{e^{\imath \theta}}$. Then, simply, we have

$$
\begin{aligned}
& \lambda T_{e^{-\imath \theta}} H X_{1}=\lambda H T_{e^{\imath \theta}} X_{1}=H\left(\lambda T_{e^{\imath \theta}} X_{1}\right)=H X_{1} T_{e^{\imath \theta}}, \\
& T_{e^{-\imath \theta}} X_{2} H=\lambda X_{2} T_{e^{-\imath \theta}} H=\lambda X_{2} T_{e^{-\imath \theta}} H=\lambda X_{2} H T_{e^{\imath \theta}},
\end{aligned}
$$

proving the assertion.

\section{Conflict of Interests}

The author declares that there is no conflict of interests regarding the publication of this paper. 


\section{Acknowledgment}

The author would like to express his sincere gratitude to the anonymous referee for his/her helpful comments that will help improve the quality of the paper.

\section{References}

[1] P. L. Duren, Theory of $\mathrm{H}^{p}$ spaces, Pure and Applied Mathematics, Vol. 38, Academic Press, New York, NY, USA, 1970.

[2] W. Rudin, Real and Complex Analysis, McGraw-Hill, 3rd edition, 1987.

[3] A. Böttcher and B. Silbermann, Analysis of Toeplitz Operators, Springer, Berlin, Germany, 1990.

[4] A. Böttcher and B. Silbermann, Introduction to large Truncated Toeplitz Matrices, Springer, New York, NY, USA, 1999.

[5] R. G. Douglas, Banach Algebra Techniques in Operator Theory, Academic Press, New York, NY, USA, 1972.

[6] R. G. Douglas, Banach Algebra Techniques in the Theory of Toeplitz Operators, vol. 15 of CBMS Regional Conference Series in Mathematics, American Mathematical Society, Providence, RI, USA, 1973.

[7] I. Gohberg and N. Krupnik, One-Dimensional Linear Singular Integral Equations, vol. 1, 2, Birkhäuser, Basel, Switzerland, 1992.

[8] N. K. Nikolski, Treatise on the Shift Operator, Springer, Berlin, Germany, 1986.

[9] R. G. Douglas, "On the operator equation $S^{*} X T=X$ and related topics," Acta Scientiarum Mathematicarum (Szeged), vol. 30, pp. 19-32, 1969.

[10] V. Pták, "Factorization of Toeplitz and Hankel operators," Mathematica Bohemica, vol. 122, no. 2, pp. 131-145, 1997.

[11] J. Barría and P. R. Halmos, "Asymptotic toeplitz operators," Transactions of the American Mathematical Society, vol. 273, no. 2, pp. 621-630, 1982.

[12] S. Sun, “On the operator equation $U^{*} T U=\lambda T$," Kexue Tongbao, vol. 29, pp. 298-299, 1984.

[13] A. Brown and P. R. Halmos, "Algebraic properties of Toeplitz operators," Journal für die Reine und Angewandte Mathematik, vol. 213, pp. 89-102, 1964.

[14] R. A. Martinez-Avendano, "A generalization of Hankel operators," Journal of Functional Analysis, vol. 190, pp. 418-446, 2002.

[15] A. Lambert and S. Petrovic, "Beyond hyperinvariance for compact operators," Journal of Functional Analysis, vol. 219, no. 1, pp. 93-108, 2005.

[16] S. Brown, "Connections between an operator and a compact operator that yield hyperinvariant subspaces," Journal of Operator Theory, vol. 1, no. 1, pp. 117-121, 1979.

[17] H. W. Kim, R. Moore, and C. M. Pearcy, "A variation of Lomonosov's theorem," Journal of Operator Theory, vol. 2, no. 1, pp. 131-140, 1979.

[18] V. I. Lomonosov, "Invariant subspaces of the family of operators that commute with a completely continuous operator," Funkcional' nyi Analiz i ego Prilozenija, vol. 7, no. 3, pp. 55-56, 1973 (Russian). 


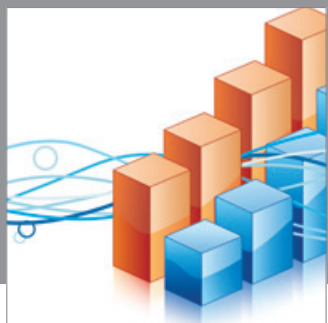

Advances in

Operations Research

mansans

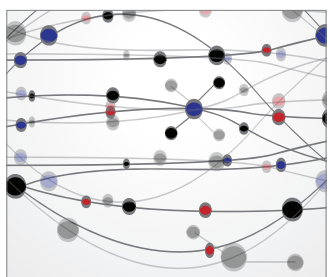

The Scientific World Journal
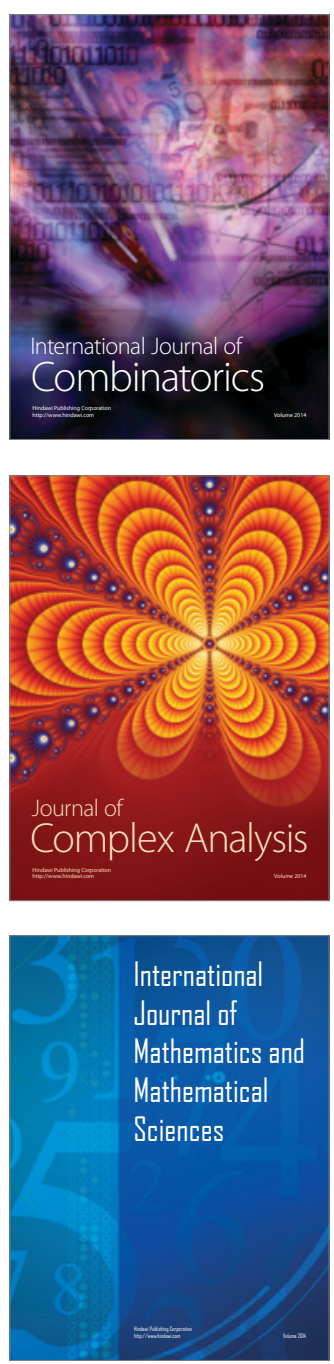
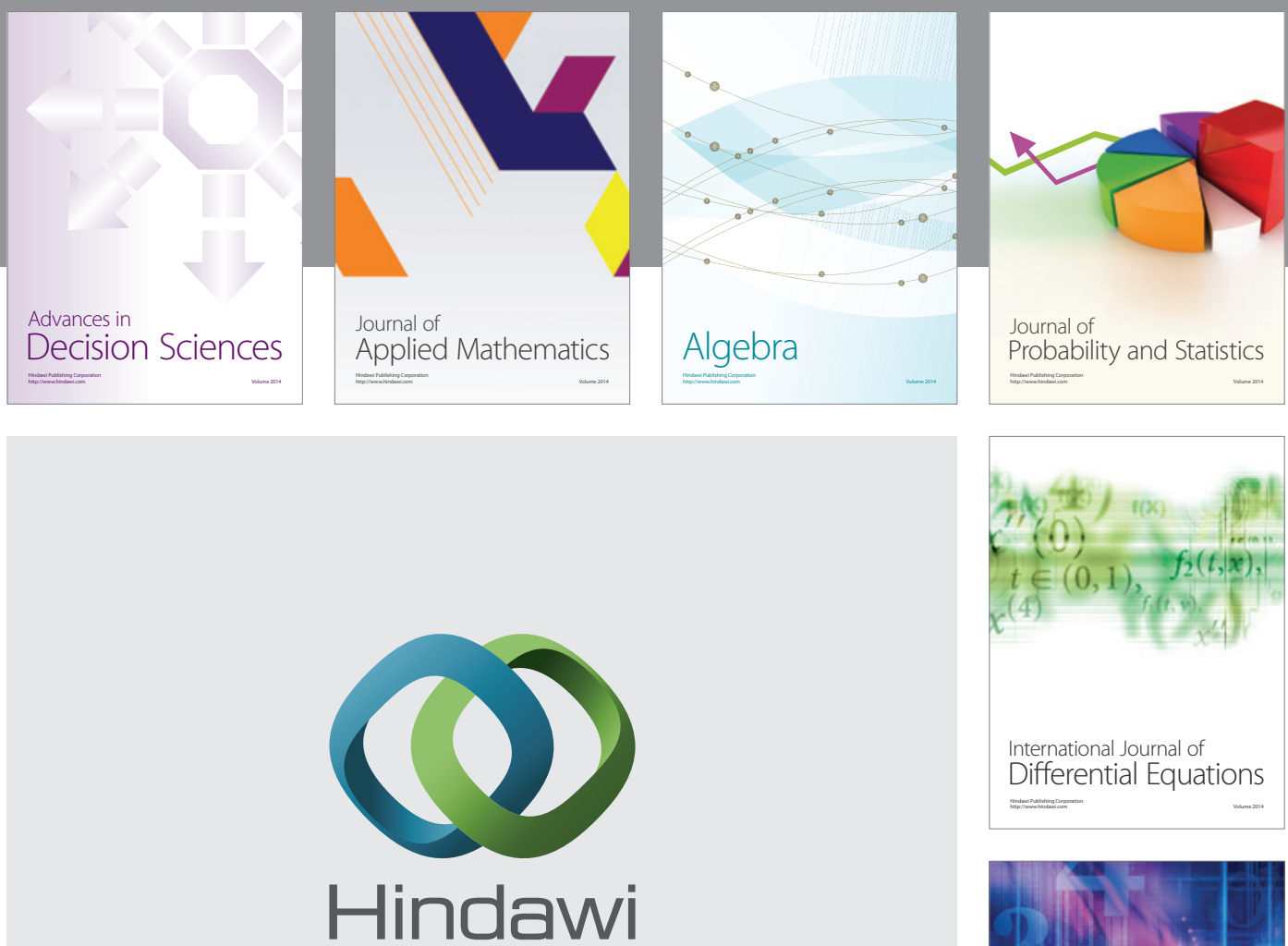

Submit your manuscripts at http://www.hindawi.com
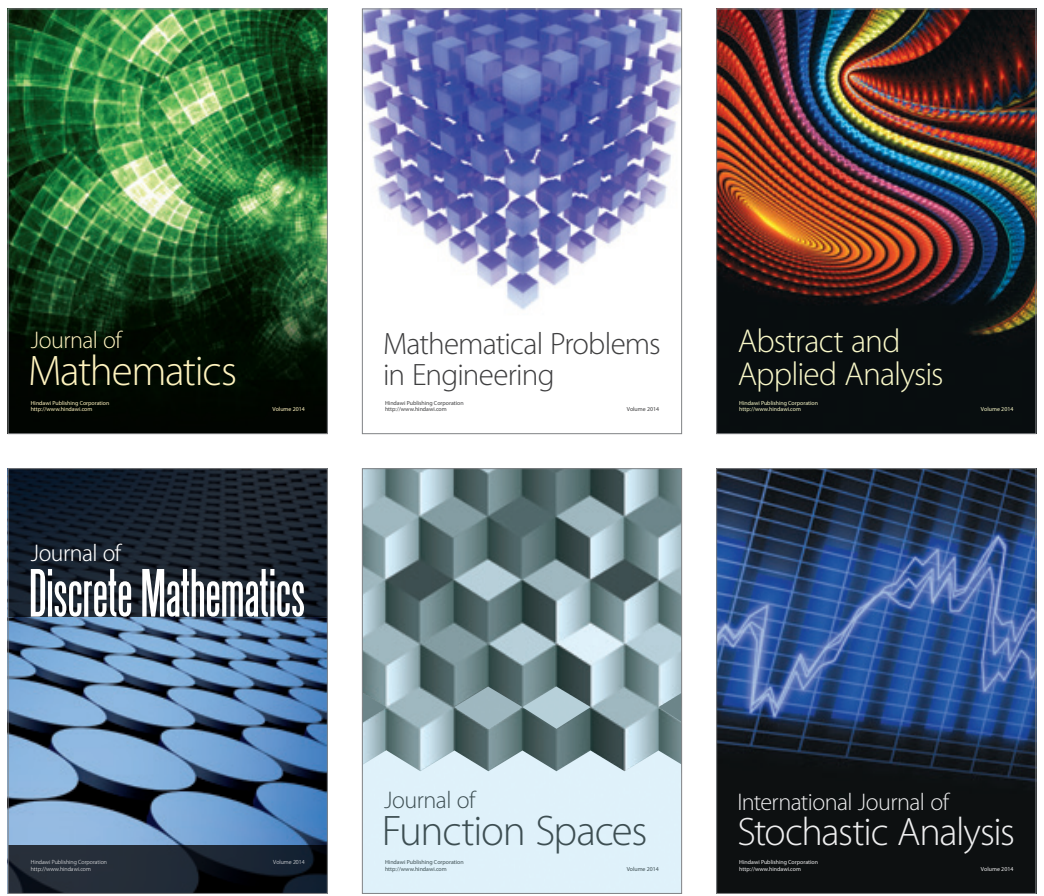

Journal of

Function Spaces

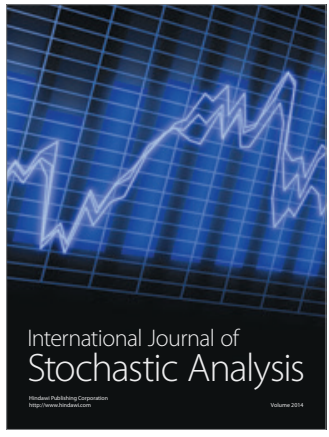

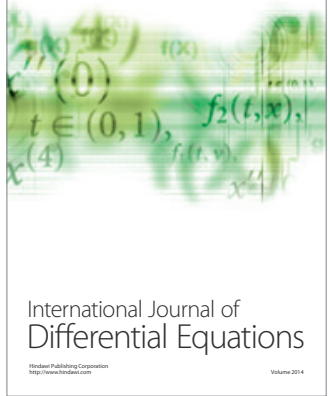
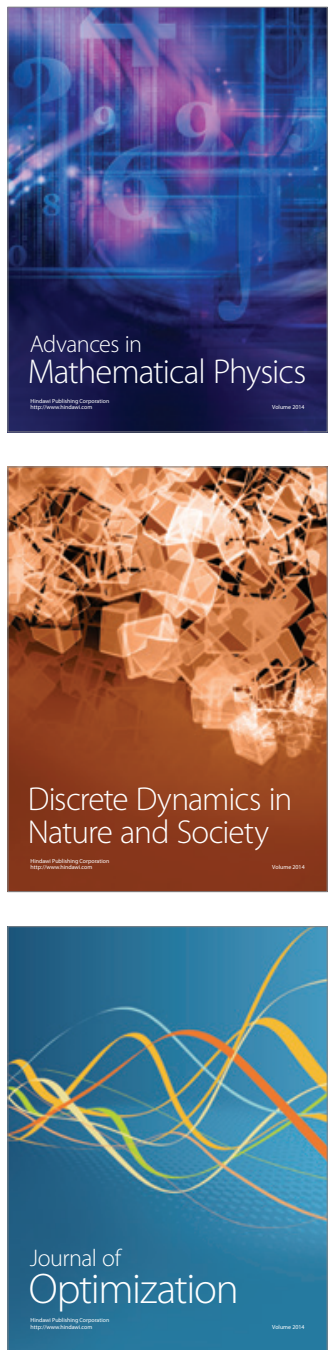